\title{
Application of Pulsed Rhythmic Drug Administration to Ovulation Induction Therapy in PCOS Patients with Clomiphene-Resistance: a Retrospective Research
}

\author{
Xinyue Zhang ${ }^{1} \cdot$ Aiyan Zheng ${ }^{2} \cdot$ Jihong Yang ${ }^{1} \cdot$ Ting Feng $^{1} \cdot$ Yan Zhang ${ }^{1} \cdot$ Yingying Hao $^{1} \cdot$ Suying $\mathrm{Li}^{1} \cdot$ Yun Qian $^{1}$ (D)
}

Received: 3 November 2020 / Accepted: 25 May 2021 / Published online: 3 June 2021

(C) The Author(s) 2021

\begin{abstract}
There is currently a dispute over the choice of ovulation induction treatment for infertile women with polycystic ovary syndrome (PCOS). The objective of this study is to compare the therapeutic effect of pulsed rhythmic administration protocol (PRAP) with conventional letrozole + human menopausal gonadotropin $(\mathrm{HMG})$ in patients with clomiphene-resistance polycystic ovary syndrome (PCOS). A retrospective analysis of 821 intrauterine insemination (IUI) cycles between January 2015 and January 2020 was performed. Of these, 483 cycles were treated with a pulsed rhythmic administration protocol (PRAP), and 338 cycles were treated with conventional letrozole $+\mathrm{HMG}$ protocol (LHP). The therapeutic effect of the two protocols has been compared. The pregnancy rate was $18.07 \%$ in the LHP and $27.07 \%$ in the PRAP. The ongoing pregnancy rate in LHP was $14.46 \%$ and in PRAP was $22.73 \%$. The research suggests that PRAP is more effective than LHP and could be an adequate ovulation induction strategy for the IUI cycle of patients with clomiphene-resistance PCOS.
\end{abstract}

Keywords Pulsed rhythmic administration $\cdot$ Polycystic ovary syndrome $\cdot$ Intrauterine insemination

(polycystic ovary) [3]. Currently, there is no cure for PCOS, and the treatment focuses on relieving symptoms and emphasizing a healthy lifestyle to minimize the risk of complications [4].

\section{Introduction}

Polycystic ovary syndrome (PCOS) is a common endocrine disease in women of childbearing age, and it is the main cause of female infertility worldwide [1]. It may contribute to many adverse reproductive, metabolic, and psychological effects, with unknown etiology, which may be related to environmental factors, genetic conditions, and intrauterine exposure [2]. Polycystic ovary syndrome is characterized by a disturbed menstrual cycle (oligomenorrhea or amenorrhea), elevated androgen levels (hyperandrogenism), and multiple ovarian cysts

Xinyue Zhang and Aiyan Zheng are regarded as joint first authors.

Yun Qian

qianyun@njmu.edu.cn

1 Reproductive Medical Center of the Second Affiliated Hospital of Nanjing Medical University, Nanjing 210011, China

2 Center for Reproduction and Genetics, Suzhou Municipal Hospital, the Affiliated Suzhou Hospital of Nanjing Medical University, Suzhou 215002, China
Intrauterine insemination (IUI) is widely used to improve the chances of pregnancy. It is simple, easy to manage, and relatively low cost, especially for patients with unexplained or mild male infertility [5]. Lifestyle change such as exercise and weight loss in overweight women is considered the first treatment for women with PCOS [6]. However, in the reproductive field, infertility caused by chronic anovulation is the most common reason for women with PCOS to seek treatment [7]. Among these women, ovulation induction is the better choice; thus, CC used to be the first-line treatment; letrozole should be the first-line treatment according to new guidelines [8-10]. However, clinical evidence indicates that although the ovulation rate for patients is higher after ovulation induction, the clinical pregnancy rate is not satisfactory [11]. At the same time, $\sim 20 \%$ women with PCOS are resistant to CC, they remains anovulatory under $\mathrm{CC}$ induction after three cycles, and they could be offered the therapy of low-dose gonadotropin [8]. Simultaneous implementation of ovulation induction and IUI is one of the most common solutions. Gonadotropins are used in IUI [12]; however, the use of exogenous gonadotropin increases the risk of multiple pregnancies; intense monitoring of ovarian response is necessary [12]. 
There is currently a dispute over the choice of ovulation induction treatment for infertile women with PCOS. Previous literature has shown that ovulation induction schemes with single or combined drugs have their advantages and disadvantages and are capable of achieving different degrees of clinical efficacy $[9,13-16]$. In the stair-step protocol, if the response is not sufficient, the dosage should be increased immediately [17], resulting in a multiple pregnancy rate almost ten times that of natural delivery [18]. In the low-dose step-up protocol, the frequency of extreme ovarian hyperstimulation is 4.6$11.0 \%$, while the multiple pregnancy rate reaches $33 \%$ [19]. $\mathrm{Xi}$ et al. found that compared with HMG alone, letrozole or $\mathrm{CC}$ combined with HMG reduced the HMG dose and the duration of treatment and the letrozole + HMG group achieved the highest rate of monofollicular development and reduced the risks of hyperstimulation for ovarian induction [15]. The final objective for women with PCOS is to achieve unifollicular cycles without causing OHSS and multiple pregnancies [19]. In the long-term clinical practice of the Reproductive Medicine Center of the Second Affiliated Hospital of Nanjing Medical University, pulsed rhythmic administration protocol (PRAP) has been shown to have achieved a successful pregnancy rate in the ovulation induction in infertile PCOS women; they had unsuccessful ovulation induction treatment with $\mathrm{CC}$ after three cycles. In this study, we retrospectively analyzed conventional letrozole + human menopausal gonadotropin (HMG) and PRAP to provide a new and appropriate regimen for ovulation induction in patients with clomiphene-resistance PCOS.

\section{Material and Methods}

\section{Research Subjects}

For retrospective analysis, clomiphene-resistance PCOS patients undergoing IUI-assisted pregnancy at the Reproductive Medicine Center of the Second Affiliated Hospital of Nanjing Medical University from January 2015 to January 2020 were collected. The screening process is shown in detail in Fig. 1. There were 785 PCOS patients with clomiphene-resistance, among which 537 patients underwent 833 IUI cycles, and the others underwent outpatient induction. The cycle cancellation rate was $1.44 \%$. Data were obtained from the medical records received from patients. The study was approved by the ethics committee of the medical institution. Every participant has signed informed consent for the study. All women met the following criteria: (1) According to the Rotterdam Consensus, they have been classified as PCOS [20]; (2) females had been infertile for more than 1 year; (3) females were 21-37 years of age; (4) females had CC resistance after three cycles; (5) females had at least one fallopian tube unobstructed by salpingography or laparoscopy or hysteroscopy; (6) males had at least two semen tests before IUI, excluding azoospermia and severe oligospermia. Exclusion criteria were as follows: (1) the female has a history of other endocrine diseases, such as hyperprolactinemia, thyroid dysfunction or diabetes, tumor history, and family tumor history; (2) B-ultrasonography of the female had intrauterine adhesions, endometrial polyps, and uterine malformations; (3) she has premature ovarian failure; (4) chromosomal abnormalities were present in one or both sides of the couple. After consulting infertility treatment and IUI surgery, induction programs were suggested to all participants. Patients were divided into two groups based on the professional judgment of the experts and the wishes of the patients. The two induction programs have been compared, while the baseline characteristics were similar in both groups.

\section{Ovarian Induction Programs}

\section{Letrozole + HMG Protocol (LHP)}

Letrozole was taken one to two pills daily from day 3 to day 7 of the menstrual cycle in women treated with LHP $(5 \mathrm{mg}$ letrozole was used for patients with BMI >40) [21]. Meanwhile, from day 3 of menstruation to the day before human chorionic gonadotropin (hCG) triggering, HMG 75225IU was administered by injection every other day. Ultrasound examination was performed on day 9 of the menstrual cycle to examine the number and size of follicles and endometrial thickness. From this stage on, the dose of HMG was adjusted according to the individual response of each patient. When one or two follicles were $18 \mathrm{~mm}$ in diameter, hCG (10000 IU) was injected intramuscularly to trigger oocyte maturation.

\section{Pulsed Rhythmic Administration Protocol (PRAP)}

One or two pills of letrozole were taken every day from day 3 to 7 of the menstrual cycle (5mg letrozole was used for patients with BMI >40) [21]. Meanwhile, follicle-stimulating hormone (FSH) was given on days 3,6 , and 8 of menstruation with an initial dose of 75 IU. Ultrasound examination was performed on day 9 . When the follicle diameter was more than $10 \mathrm{~mm}$, the same dose of HMG was used until the follicle matures. If no significant follicular growth was observed on day 9, the dosing interval was shortened, and FSH was changed to injected every other day or daily; without any change in dose, it was still the initial 75IU. When the interval of administration was shortened and the follicle diameter was more than $10 \mathrm{~mm}$, the same dose of HMG was used until the follicle matures. If there was no dominant follicular development in this cycle after the administration interval was shortened and FSH injection was continued for more than day 16, the cycle will be cancelled and the initial dose of FSH in the 
Fig. 1 Flow chart of clomipheneresistance PCOS patients' inclusion in the Reproductive Medical Center of the Second Affiliated Hospital of Nanjing Medical University. np: number of patients, nc: number of cycles

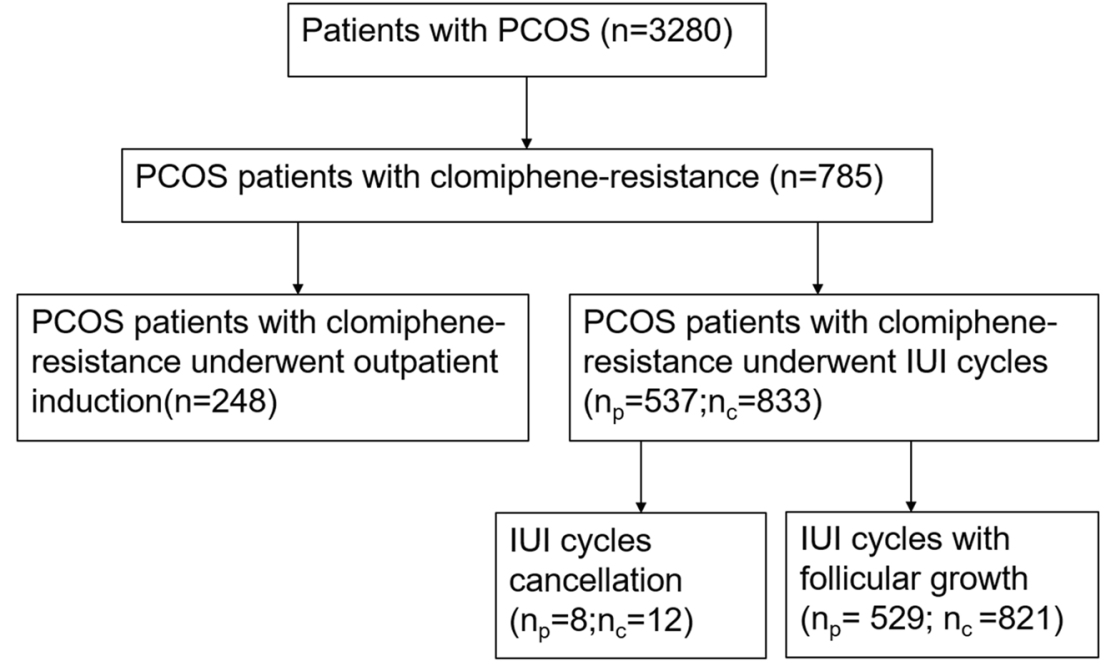

next cycle will be increased to $150 \mathrm{IU}$; the interval was maintained with the same pattern of administration rhythm. If there was still no dominant follicular development, the initial dose of FSH in the next cycle will be increased to $225 \mathrm{IU}$ (this is the maximum dose). The detail process is shown in Fig. 2.

\section{Intrauterine Insemination (IUI) Technique}

Semen samples were collected according to WHO standards, while abstinence was needed for 3-5 days before IUI was performed. Semen was collected into a sterile semen cup by a masturbation and liquefied at room temperature for $30 \mathrm{~min}$. The semen was treated with a gradient centrifugation method, and routine examinations were carried out before and after treatment. If the total amount of the male forward motile sperm was not less than $10 \times 10^{6} / \mathrm{ml}$ after the procedure, the treated semen was slowly injected into the woman's uterine cavity along the direction of the cervix, and the female rested for $30 \mathrm{~min}$ after the operation.

\section{Luteal Support and Postoperative Follow-Up}

Luteal support was given after ovulation by oral administration of dydrogesterone $40 \mathrm{mg} /$ day for 14 days. After 14 days, blood or urine $\mathrm{HCG}$ was measured. If urine $\mathrm{HCG}$ was positive or blood $\mathrm{HCG} \geq 50 \mathrm{mIU} / \mathrm{ml}$, it was identified as biochemical pregnancy. If the pregnancy sac was found by transvaginal ultrasound, it was diagnosed as a clinical pregnancy. The ongoing pregnancy rate was defined as the percentage of women with ongoing pregnancy $\geq 12$ weeks [22].

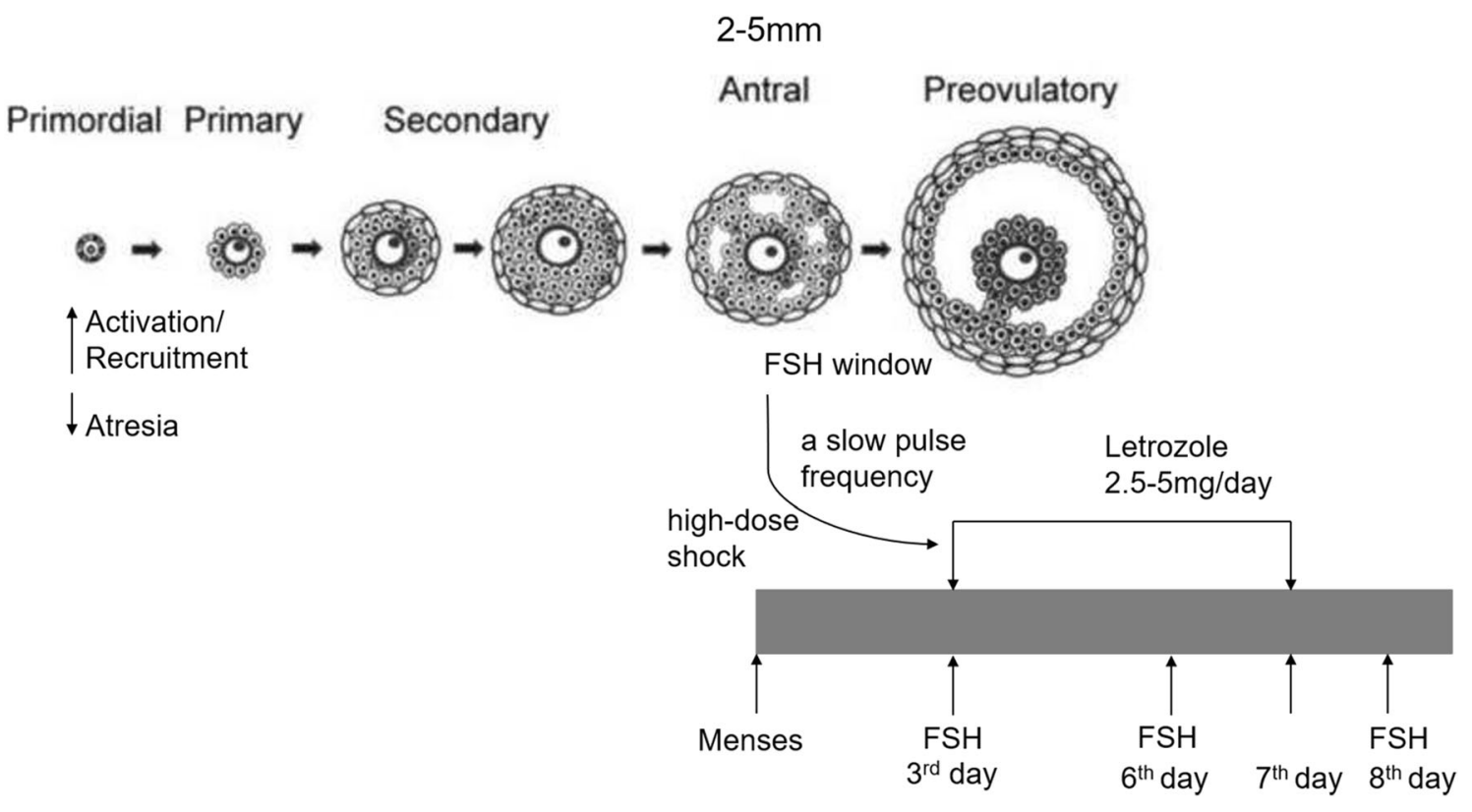

Fig. 2 The pulsed rhythmic administration protocol 


\section{Statistical Analysis}

SPSS 18.0 software (SPSS, USA) has been used for statistical analysis. The data were expressed as mean \pm standard deviation and analyzed by t-test (The data were normally distributed after analyzed.). The ovulation rate and pregnancy outcome were analyzed by the chi-square test or Fisher exact test. P < 0.05 was statistically significant.

\section{Results}

The number of ovulation cycles was 249 in the LHP group, and there were 374 ovulation cycles in the PRAP group. The ovulation rate in the LHP group was $77.43 \%$ and in the PRAP group was $73.67 \%$; there were no differences between the two groups $(\mathrm{P}=0.215)$. Of the 623 ovulation cycles, there were 374 cycles and 211 participants in the PRAP group and 249 cycles and 141 participants in the LHP group, with no moderate or severe ovarian hyperstimulation. Both groups had relatively many patients with primary infertility. There were four patients in the PRAP group and two patients in the LHP group used letrozole in a dose of $5 \mathrm{mg}$. The baseline characteristics of patients in the two groups are shown in Table 1. The mean age of female patients in the PRAP group was $27.71 \pm 3.10$ years, and that of the LHP group was $27.39 \pm 3.38$ years. There was no significant difference in female age, male age, and infertility duration. Body mass index (BMI) was $25.07 \pm 4.51$ in the PRAP group and $25.21 \pm 4.55$ in the LHP group. The basal antral follicle count (AFC) was $25.14 \pm 6.97$ in the PRAP group and $24.66 \pm 6.82$ in the LHP group. The baseline characteristics of both groups were similar, which means that the two sets of data were comparable.

Table 2 shows the levels of essential sex hormones. Serum FSH was $6.54 \pm 2.22$ in the PRAP group and $6.61 \pm 1.82$ in

Table 1 Baseline characteristics of the letrozole + HMG protocol (LHP) and the pulsed rhythmic administration protocol (PRAP)

\begin{tabular}{llll}
\hline Parameters & LHP & PRAP & P \\
\hline Number of cycles & 338 & 483 & NA \\
Rate of ovulation (\%) & $73.67(249 / 338)$ & $77.43(374 / 483)$ & 0.215 \\
Number of participants & 141 & 211 & NA \\
Primary infertility & 182 & 284 & NA \\
Secondary infertility & 67 & 90 & NA \\
Age, female, years & $27.39 \pm 3.38$ & $27.71 \pm 3.10$ & 0.235 \\
Age, male, years & $28.80 \pm 3.85$ & $28.82 \pm 3.30$ & 0.960 \\
Duration of infertility years & $2.90 \pm 1.79$ & $2.80 \pm 1.78$ & 0.523 \\
BMI & $25.21 \pm 4.55$ & $25.07 \pm 4.51$ & 0.697 \\
Basal AFC & $24.66 \pm 6.82$ & $25.14 \pm 6.97$ & 0.394 \\
\hline
\end{tabular}

Data are expressed as mean $\pm \mathrm{SD}$. $\mathrm{P}<0.05$ significantly different from control group the LHP group. Meanwhile, serum luteinizing hormone (LH) was $5.06 \pm 2.95$ in the PRAP group while $6.41 \pm 3.27$ in the LHP group. The serum LH level in each group was lower than that of FSH, and there was no difference in serum testosterone levels, suggesting that the endocrine status of clomipheneresistance PCOS patients was normal and suitable for ovulation induction therapy. There was no significant difference in serum estradiol, prolactin (PRL), and progesterone levels.

As shown in Table 3, the total dose of FSH/LH in the PRAP group was $953.24 \pm 483.22 \mathrm{IU}$, and that in the LHP group was $798.39 \pm 559.35$ IU. The duration of induction in the PRAP group was $5.39 \pm 2.00$ days, which was shorter than that in the LHP group. The final cost of LHP group was 804.13 \pm 823.49 , and the PRAP group was $887.83 \pm 469.74(\mathrm{P}=0.147)$. There was no significant difference between the two groups in the number of dominant follicles and endometrial thickness on the day before IUI surgery. Neither group had moderate or severe ovarian hyperstimulation. Biochemical pregnancy and clinical pregnancy in the PRAP group were higher than those in the LHP group. Among pregnant women, there were eighteen twin pregnancies in the PRAP group and six twin pregnancies in the LHP group. There was one case of ectopic pregnancy in the LHP group. However, four patients in the PRAP group underwent multiple pregnancy reduction. There was no significant difference in the abortion rate between the two groups. The ongoing pregnancy rate in the PRAP group was higher than that in the LHP group.

\section{Discussion}

\section{Main Results}

Present retrospective analysis suggests that PRAP may be a useful stimulus protocol for women with clomipheneresistance PCOS. On the basis of not significantly increasing the final cost of treatment, the ongoing pregnancy rates in the PRAP group have increased significantly relative to the LHP group.

Table 2 Basal hormone levels between the letrozole + HMG protocol (LHP) and the pulsed rhythmic administration protocol (PRAP)

\begin{tabular}{llll}
\hline Parameters & LHP & PRAP & P \\
\hline Serum FSH & $6.61 \pm 1.82$ & $6.54 \pm 2.22$ & 0.761 \\
Serum LH & $6.41 \pm 3.27$ & $5.06 \pm 2.95$ & 0.000 \\
Serum E2 & $47.75 \pm 27.54$ & $54.87 \pm 64.57$ & 0.294 \\
Serum T & $0.57 \pm 0.24$ & $0.56 \pm 0.22$ & 0.527 \\
Serum PRL & $11.69 \pm 5.15$ & $12.35 \pm 5.72$ & 0.532 \\
Serum P & $0.62 \pm 0.18$ & $0.89 \pm 0.53$ & 0.134 \\
\hline
\end{tabular}

Data are expressed as mean $\pm \mathrm{SD} . \mathrm{P}<0.05$ significantly different from control group 
Table 3 Clinical characteristics of the letrozole + HMG protocol (LHP) and the pulsed rhythmic administration protocol (PRAP)

\begin{tabular}{llll}
\hline Parameters & LHP & PRAP \\
\hline Total dose of FSH/LH (IU) & $798.39 \pm 559.35$ & $953.24 \pm 483.22$ & 0.000 \\
Duration of induction (days) & $7.75 \pm 4.63$ & $5.39 \pm 2.00$ & 0.000 \\
The final cost (RMB) & $804.13 \pm 823.49$ & $887.83 \pm 469.74$ & 0.147 \\
Number of dominant follicles & $1.20 \pm 0.47$ & $1.18 \pm 0.50$ & 0.540 \\
Endometrial thickness on the day before IUI (cm) & $9.09 \pm 2.32$ & $9.11 \pm 2.00$ & 0.908 \\
The incidence of OHSS (\%) & 0 & $110(29.41)$ & NA \\
Biochemical pregnancy (\%) & $54(21.67)$ & $101(27.01)$ & 0.032 \\
Clinical pregnancy (\%) & $45(18.07)$ & $18(4.81)$ & 0.010 \\
Twin pregnancies (\%) & $6(2.19)$ & $16(4.28)$ & 0.127 \\
Miscarriage $(\%)$ & $9(3.61)$ & $85(22.73)$ \\
Ongoing pregnancy $\geq 12$ weeks, no. of women $(\%)$ & $36(14.46)$ & 0.679 \\
\hline
\end{tabular}

Data are expressed as mean $\pm \mathrm{SD}$. $\mathrm{P}<0.05$ significantly different from control group

\section{Interpretation of Results}

In women with PCOS, pulsation of gonadotropin-releasing hormone $(\mathrm{GnRH})$ is usually increased, resulting in an increase in the LH released by the pituitary gland and the LH/FSH ratio [18]. PCOS-associated ovarian defects are affected by various internal and external ovarian factors, which affect follicular development in multiple stages, causing increased recruitment, failure to gain dominance, or atresia, leading to antral follicular developmental stagnation [23]. Most of the phenotypes of anovulatory PCOS are associated with the 2-8 mm antral follicles accumulation [24]. Since patients with PCOS typically have high LH and low FSH levels, estrogen receptor antagonists or aromatase inhibitors are used to reduce the negative feedback of estrogen on pituitary FSH secretion, or exogenous FSH is used to assist ovulation [25]. Therefore, letrozole and exogenous FSH were used to promote ovulation in both the schemes in this study.

During adolescence, the maturation of the hypothalamuspituitary-gonad (HPG) axis contributes to the pulsed release of FSH and LH in the pituitary gland, resulting in the development of antral follicular cycle $\geq 2 \mathrm{~mm}$, and the initiation of ovulation and menstrual cycle [26]. FSH is a vital nutrient factor affecting follicular cell proliferation, survival, and periodic recruitment of antral follicles. Follicular development can be divided into gonadotropin-dependent and gonadotropinreactive stages. Exogenous gonadotropin therapy is likely to promote the growth of large antral follicles and smaller preantral follicles [27]. During the physiological human menstrual cycle, a slight increase in serum FSH level $(\sim 30 \%)$ is enough to initiate the growth of the most sensitive ovarian follicles, because the negative feedback of estradiol is reduced throughout the follicular period [28]. Along with follicular growth, they produce estradiol, which provides negative feedback on FSH secretion, thereby limiting the "FSH window"- the period during which FSH levels are held above the threshold to prevent non-dominant follicles atresia [29]. There is differential sensitivity between individual antral follicles in the cohort, and single follicle growth can be successfully induced in the ovulation induction process by gradually reaching the lowest threshold of the most sensitive follicle [30].

In hyperandrogenemia women destined to develop PCOS, this nocturnal increase in ovarian steroids may not be sufficient to inhibit the GnRH pulse generators, resulting in sustained and rapid release of LH, impaired FSH production, and follicular dysplasia. In vivo studies have shown that the faster the frequency of pulsatile exogenous $\mathrm{GnRH}$, the more favorable to the release of LH. Nevertheless, the slow pulse rate was beneficial to the secretion of $\mathrm{FSH}$, which increased serum FSH and decreased serum LH [31]. Therefore, in this research, in the PRAP, FSH was administered on days 3, 6, and 8 of menstruation instead of every other day to facilitate the establishment of a slow pulse frequency and facilitate the secretion of FSH. The role of FSH in follicular development is limited to the early stages, including follicular recruitment and selection. FSH levels in early follicles play an essential role in the final stages of follicular development in the human menstrual cycle. Controlled recruitment of dominant follicles can be achieved by inducing high levels of FSH in the blood within a short period of time during the early stages of the follicular phase [32]. In this study, PRAP had a shorter duration of induction and a higher dose of FSH/LH, as compared to LHP. At the early stage of follicular development, the dominant follicles were established by the high-dose shock. Dominant follicles must remain unique responsiveness and escape the consequences of FSH suppression induced by their own accelerated of estrogen production. The proliferation rate of the granules exceeded its cohort, which makes the dominant follicle to have a higher content of the FSH receptor. On day 9 of the cycle, after ablation of dominant follicles, no other 
follicles remain fully developed to replace and enable ovulation to occur on time [33]. When the dominant follicle reaches a diameter of $10 \mathrm{~mm}$, divergence occurs [26]. Once the follicle diameter exceeds $10 \mathrm{~mm}$, LH may stimulate the function of granulocyte, and during the selection process, the dependence of dominant follicles on FSH decreases, and the response to LH increases [34]. Therefore, in PRAP, when the follicle diameter is greater than $10 \mathrm{~mm}$, the drug is replaced by the same dose of HMG. PRAP improves both clinical and ongoing pregnancy rates.

\section{Advantages and Limitations}

The advantage of this study lies in its larger sample size compared with other similar studies, and we proposed a new IUI induction protocol for patients with clomiphene-resistance PCOS that may have a significant reference. This study has also observed a few limitations. This is a retrospective study with a short duration and limited types of induction protocols. For patients with clomiphene-resistance PCOS, the ideal treatment has not been determined. Also, the allocation of the subjects was based on the patient's wishes or the suggestion of the assistant doctor, which allowed for the introduction of biases that may confound the analysis. Therefore, well-designed prospective randomized controlled clinical trials are needed in the future to compare various ovarian induction protocols further.

\section{Conclusion}

PRAP may be the right choice for clomiphene-resistance PCOS patients undergoing the IUI cycle. On the basis of not significantly increasing the final cost of treatment, it can obtain a higher pregnancy rate and ongoing pregnancy rate. However, further prospective clinical trials are required to provide more substantial evidence.

Abbreviations $H M G$, Human menopausal gonadotropin; PCOS, Polycystic ovary syndrome; IUI, Intrauterine insemination; PRAP, Pulsed rhythmic administration protocol; $L H P$, Letrozole + HMG proto$\mathrm{col}$; $C O C$, Combined oral contraceptive; $h C G$, human Chorionic gonadotropin; FSH, Follicle-stimulating hormone; BMI, Body mass index; $A F C$, Antral follicle count; $L H$, Luteinizing hormone; $P R L$, prolactin; $G n R H$, Gonadotropin-releasing hormone; $H P G$, Hypothalamus-pituitary-gonad

Author Contribution $\mathrm{XYZ}$ and $\mathrm{AYZ}$ contributed to the data collection and interpretation and the initial draft of the manuscript. JHY carried out the IUI technique and contributed to the draft and revision of the manuscript. TF carried out the semen analysis and processing. YZ contributed to the data collection and analysis. YYH contributed to the data collection and analysis. SYL carried out the semen processing. YQ design of the study and the patient recruitment and critically revised the manuscript. All authors provided a critical review and approved the final manuscript.
Availability of Data and Materials The datasets used and/or analyzed during the current study are available from the corresponding author on reasonable request.

\section{Declarations}

Ethics Approval and Consent to Participate The study was approved by the Reproductive Medicine Ethics Committee of the Second Affiliated Hospital of Nanjing Medical University (date: October 9, 2014). All participants were informed of the three induction protocols after counseling for infertility treatments and IUI procedures.

Consent for Publication Not applicable

Competing Interests The authors declare no competing interests.

Open Access This article is licensed under a Creative Commons Attribution 4.0 International License, which permits use, sharing, adaptation, distribution and reproduction in any medium or format, as long as you give appropriate credit to the original author(s) and the source, provide a link to the Creative Commons licence, and indicate if changes were made. The images or other third party material in this article are included in the article's Creative Commons licence, unless indicated otherwise in a credit line to the material. If material is not included in the article's Creative Commons licence and your intended use is not permitted by statutory regulation or exceeds the permitted use, you will need to obtain permission directly from the copyright holder. To view a copy of this licence, visit http://creativecommons.org/licenses/by/4.0/.

\section{References}

1. Davinelli S, Nicolosi D, Di Cesare C, Scapagnini G, Di Marco R. Targeting metabolic consequences of insulin resistance in polycystic ovary syndrome by D-chiro-inositol and emerging nutraceuticals: a focused review. Journal of clinical medicine. 2020;9(4). https://doi.org/10.3390/jcm9040987.

2. Copp T, Muscat DM, Hersch J, McCaffery KJ, Doust J, Mol BW, et al. Clinicians' perspectives on diagnosing polycystic ovary syndrome in Australia: a qualitative study. Human reproduction (Oxford, England). 2020;35(3):660-8. https://doi.org/10.1093/ humrep/deaa005.

3. Zhang Y, Ho K, Keaton JM, Hartzel DN, Day F, Justice AE, et al. A genome-wide association study of polycystic ovary syndrome identified from electronic health records. American journal of obstetrics and gynecology. 2020;223:559.e1-559.e21. https://doi.org/10. 1016/j.ajog.2020.04.004.

4. Teede HJ, Misso ML, Boyle JA, Garad RM, McAllister V, Downes $\mathrm{L}$, et al. Translation and implementation of the Australian-led PCOS guideline: clinical summary and translation resources from the International Evidence-based Guideline for the Assessment and Management of Polycystic Ovary Syndrome. The Medical journal of Australia. 2018;209(S7):S3-s8.

5. Huang S, Wang R, Li R, Wang H, Qiao J, Mol BWJ. Ovarian stimulation in infertile women treated with the use of intrauterine insemination: a cohort study from China. Fertility and sterility. 2018;109(5):872-8. https://doi.org/10.1016/j.fertnstert.2018.01.008.

6. Consensus on infertility treatment related to polycystic ovary syndrome. Human reproduction (Oxford, England). 2008;23(3):46277. https://doi.org/10.1093/humrep/dem426.

7. Weiss NS, Kostova E, Nahuis M, BWJ M, Van der Veen F, Van Wely M. Gonadotrophins for ovulation induction in women with polycystic ovary syndrome. The Cochrane database of systematic 
reviews. 2019;1:Cd010290. https://doi.org/10.1002/14651858. CD010290.pub3.

8. Balen AH, Morley LC, Misso M, Franks S, Legro RS, Wijeyaratne $\mathrm{CN}$, et al. The management of anovulatory infertility in women with polycystic ovary syndrome: an analysis of the evidence to support the development of global WHO guidance. Human reproduction update. 2016;22(6):687-708. https://doi.org/10.1093/ humupd/dmw025.

9. Wang R, Li W, Bordewijk EM, Legro RS, Zhang H, Wu X, et al. First-line ovulation induction for polycystic ovary syndrome: an individual participant data meta-analysis. Human reproduction update. 2019;25(6):717-32. https://doi.org/10.1093/humupd/ dmz029.

10. Bordewijk EM, Ng KYB, Rakic L, Mol BWJ, Brown J, Crawford $\mathrm{TJ}$, et al. Laparoscopic ovarian drilling for ovulation induction in women with anovulatory polycystic ovary syndrome. The Cochrane database of systematic reviews. 2020;2(2):CD001122.

11. Rezk M, Shaheen AE, Saif E-NI. Clomiphene citrate combined with metformin versus letrozole for induction of ovulation in clomipheneresistant polycystic ovary syndrome: a randomized clinical trial. Gynecological endocrinology : the official journal of the International Society of Gynecological Endocrinology. 2018;34(4): 298-300. https://doi.org/10.1080/09513590.2017.1395838.

12. Melo AS, Ferriani RA, Navarro PA. Treatment of infertility in women with polycystic ovary syndrome: approach to clinical practice. Clinics (Sao Paulo, Brazil). 2015;70(11):765-9. https://doi. org/10.6061/clinics/2015(11)09.

13. Wang R, Kim BV, van Wely M, Johnson NP, Costello MF, Zhang H, et al. Treatment strategies for women with WHO group II anovulation: systematic review and network meta-analysis. BMJ (Clinical research ed). 2017:356:j138. https://doi.org/10.1136/bmj.j138.

14. Amer SA, Smith J, Mahran A, Fox P, Fakis A. Double-blind randomized controlled trial of letrozole versus clomiphene citrate in subfertile women with polycystic ovarian syndrome. Human reproduction (Oxford, England). 2017;32(8):1631-8. https://doi.org/10. 1093/humrep/dex227.

15. Xi W, Liu S, Mao H, Yang Y, Xue X, Lu X. Use of letrozole and clomiphene citrate combined with gonadotropins in clomipheneresistant infertile women with polycystic ovary syndrome: a prospective study. Drug design, development and therapy. 2015;9: 6001-8. https://doi.org/10.2147/dddt.S83259.

16. Ege S, Bademkiran MH, Peker N, Tahaoglu AE, Hancer Caca FN, Ozcelik SM. A comparison between a combination of letrozole and clomiphene citrate versus gonadotropins for ovulation induction in infertile patients with clomiphene citrateresistant polycystic ovary syndrome - a retrospective study. Ginekologia polska. 2020;91: 185-8. https://doi.org/10.5603/GP.a2020.0037.

17. Jones T, Ho JR, Gualtieri M, Bruno-Gaston J, Chung K, Paulson RJ, et al. Clomiphene stair-step protocol for women with polycystic ovary syndrome. Obstetrics and gynecology. 2018;131(1):91-5. https://doi.org/10.1097/aog.0000000000002418.

18. Tanbo T, Mellembakken J, Bjercke S, Ring E, Abyholm T, Fedorcsak P. Ovulation induction in polycystic ovary syndrome. Acta obstetricia et gynecologica Scandinavica. 2018;97(10):11627. https://doi.org/10.1111/aogs.13395.

19. Lan VT, Norman RJ, Nhu GH, Tuan PH, Tuong HM. Ovulation induction using low-dose step-up rFSH in Vietnamese women with polycystic ovary syndrome. Reproductive biomedicine online. 2009;18(4):516-21. https://doi.org/10.1016/s1472-6483(10) 60128-9.

20. Revised 2003 consensus on diagnostic criteria and long-term health risks related to polycystic ovary syndrome (PCOS). Human reproduction (Oxford, England). 2004;19(1):41-7. https://doi.org/10. 1093/humrep/deh098.
21. Arya S, Kupesic-Plavsic S, Mulla ZD, Dwivedi AK, Crisp Z, Jose J, et al. Ovulation induction and controlled ovarian stimulation using letrozole gonadotropin combination: a single center retrospective cohort study. Eur J Obstet Gynecol Reprod Biol. 2017;218:1238. https://doi.org/10.1016/j.ejogrb.2017.09.023.

22. Youssef MA, van Wely M, Al-Inany H, Madani T, Jahangiri N, Khodabakhshi S, et al. A mild ovarian stimulation strategy in women with poor ovarian reserve undergoing IVF: a multicenter randomized non-inferiority trial. Human reproduction (Oxford, England). 2017;32(1):112-8. https://doi.org/10.1093/humrep/ dew282.

23. Puttabyatappa M, Padmanabhan V. Ovarian and extra-ovarian mediators in the development of polycystic ovary syndrome. Journal of molecular endocrinology. 2018;61(4):R161-r84. https://doi.org/ 10.1530/jme-18-0079.

24. Legro RS. Ovulation induction in polycystic ovary syndrome: current options. Best practice \& research Clinical obstetrics \& gynaecology. 2016;37:152-9. https://doi.org/10.1016/j.bpobgyn.2016. 08.001 .

25. Jayasena CN, Franks S. The management of patients with polycystic ovary syndrome. Nature reviews Endocrinology. 2014;10(10): 624-36. https://doi.org/10.1038/nrendo.2014.102.

26. Baerwald AR, Adams GP, Pierson RA. Ovarian antral folliculogenesis during the human menstrual cycle: a review. Human reproduction update. 2012;18(1):73-91. https://doi.org/ 10.1093/humupd/dmr039.

27. McGee EA, Hsueh AJ. Initial and cyclic recruitment of ovarian follicles. Endocrine reviews. 2000;21(2):200-14. https://doi.org/ 10.1210/edrv.21.2.0394.

28. Abbara A, Patel A, Hunjan T, Clarke SA, Chia G, Eng PC, et al. FSH Requirements for follicle growth during controlled ovarian stimulation. Frontiers in endocrinology. 2019;10:579. https://doi. org/10.3389/fendo.2019.00579.

29. Hassan A, Kotb M, AwadAllah A, Wahba A, Shehata N. Follicular output rate can predict clinical pregnancy in women with unexplained infertility undergoing IVF/ICSI: a prospective cohort study. Reproductive biomedicine online. 2017;34(6):598-604. https://doi. org/10.1016/j.rbmo.2017.03.004.

30. White DM, Hardy K, Lovelock S, Franks S. Low-dose gonadotropin induction of ovulation in anovulatory women: still needed in the age of IVF. Reproduction (Cambridge, England). 2018;156(1):F1f10. https://doi.org/10.1530/rep-17-0697.

31. McCartney CR, Eagleson CA, Marshall JC. Regulation of gonadotropin secretion: implications for polycystic ovary syndrome. Seminars in reproductive medicine. 2002;20(4):317-26. https:// doi.org/10.1055/s-2002-36706.

32. Messinis IE, Templeton AA. The importance of follicle-stimulating hormone increase for folliculogenesis. Human reproduction (Oxford, England). 1990;5(2):153-6. https://doi.org/10.1093/ oxfordjournals.humrep.a137060.

33. Fritz MA, Speroff L. The endocrinology of the menstrual cycle: the interaction of folliculogenesis and neuroendocrine mechanisms. Fertility and sterility. 1982;38(5):509-29. https://doi.org/10.1016/ s0015-0282(16)46628-8.

34. Filicori M, Cognigni GE, Tabarelli C, Pocognoli P, Taraborrelli S, Spettoli D, et al. Stimulation and growth of antral ovarian follicles by selective LH activity administration in women. The Journal of clinical endocrinology and metabolism. 2002;87(3):1156-61. https://doi.org/10.1210/jcem.87.3.8322.

Publisher's Note Springer Nature remains neutral with regard to jurisdictional claims in published maps and institutional affiliations. 